TRANSACTIONS OF THE

AMERICAN MATHEMATICAL SOCIETY

Volume 361, Number 12, December 2009, Pages 6595-6611

S 0002-9947(09)04866-1

Article electronically published on July 24, 2009

\title{
GEOMETRIC INCIDENCE THEOREMS VIA FOURIER ANALYSIS
}

\author{
ALEX IOSEVICH, HADI JORATI, AND IZABELLA ŁABA
}

\begin{abstract}
We show that every non-trivial Sobolev bound for generalized Radon transforms which average functions over families of curves and surfaces yields an incidence theorem for suitably regular discrete sets of points and curves or surfaces in Euclidean space. This mechanism allows us to deduce geometric results not readily accessible by combinatorial methods.
\end{abstract}

\section{INTRODUCTION}

An incidence between a set of points $P$ and a set of curves or surfaces $\mathcal{G}$ in a Euclidean space $\mathbb{R}^{d}$ is a pair $(p, \Gamma)$, where $p \in P, \Gamma \in \mathcal{G}$, and $p$ lies on $\Gamma$. Combinatorial geometers have long been interested in bounds on the number of incidences between point sets and families of curves of a specified type. Such bounds, apart from their intrinsic interest, have found applications to a variety of other combinatorial problems, e.g., [3, [5], 23], 24]; a comprehensive survey of the area is given in [17]. A prototype result here is the classical Szemerédi-Trotter incidence theorem [29, which states that the total number of incidences $I$ between $N$ points and $M$ straight lines in the plane obeys

$$
I \lesssim N+M+(N M)^{\frac{2}{3}} .
$$

There are explicit examples showing that this bound is sharp. Here and below, $c, C$ are constants; $X \lesssim Y$ means that $X \leq C Y$ for some $C$, and $|\cdot|$ denotes the cardinality of a finite set, the Euclidean norm of a vector in $\mathbb{R}^{d}$, or the absolute value of a complex number, depending on the context. In addition, $X \lesssim Y$ means $X \leq C_{\epsilon} N^{\epsilon} Y$ for any $\epsilon>0$.

Székely [28] observed that the Szemerédi-Trotter theorem could be proved using only very limited geometrical information about straight lines, namely that a line is uniquely determined by a pair of points and that two lines can intersect at only one point. This allowed him to obtain an extension of the theorem to more general curves which satisfy similar intersection axioms.

A more substantial generalization was given by Pach and Sharir [16, who proved a version of the Szemerédi-Trotter theorem for pseudolines with $k$ degrees of freedom. The latter are defined to be a family of curves in $\mathbb{R}^{2}$ such that: (a) at most $O(1)$ curves can pass through any given $k$ points, (b) any pair of curves intersects in at most $O(1)$ points. Under these assumptions, the number of incidences between $N$

Received by the editors February 11, 2007 and, in revised form, February 4, 2008.

2000 Mathematics Subject Classification. Primary 42B35, 28 A75.

(C)2009 American Mathematical Society 
curves and $M$ points in $\mathbb{R}^{2}$ is bounded by

$$
I \lesssim M^{\frac{k}{2 k-1}} N^{\frac{2 k-2}{2 k-1}}+M+N
$$

Formulating and proving similar incidence theorems in higher dimensions turns out to be surprisingly difficult, and despite a considerable amount of interesting work in this direction, comprehensive and sharp estimates are difficult to come by. To illustrate some difficulties involved in the problem of counting incidences between points and codimension-one manifolds - henceforth surfaces - consider a family of $M$ 2-dimensional planes in $\mathbb{R}^{3}$ all containing a fixed line $L$, and a set of $N$ points all of which lie on $L$. Then the number of incidences is $M N$, since every point is incident to every line. Thus there can be no non-trivial incidence bounds for points and planes in $\mathbb{R}^{3}$, or for points and $k$-dimensional affine subspaces of $\mathbb{R}^{d}$ with $k \geq 2$, without additional assumptions. Similarly, consider a set of $N$ points in $\mathbb{R}^{4}$ supported on the unit circle in the $\left(x_{1}, x_{2}\right)$ coordinate plane, and a set of $M$ spheres of radius $\sqrt{2}$ whose centers are supported on the unit circle in the $\left(x_{3}, x_{4}\right)$ coordinate plane. Then each point lies on each sphere, and hence the number of incidences is again $M N$. Even in the "translation-invariant" setting, where the set of "surfaces" is obtained by translating a single hypersurface by elements of a fixed point set, it is still possible to construct similar examples, for instance an infinite one-dimensional family of translates of the paraboloid in $\mathbb{R}^{3}$ given by the equation $x_{3}=x_{1}^{2}+x_{2}^{2}$, all intersecting in a fixed parabola in a plane parallel to the $x_{3}$ direction.

These simple examples suggest that incidence theorems for surfaces of dimension $k>2$ in $\mathbb{R}^{d}$ must involve additional geometric assumptions, so as to preclude lowerdimensional obstructions such as those just described. Such results have indeed been obtained by combinatorial methods. This was done, for instance, in [5], 25], 24] for spheres, in [6, 24] for $k$-dimensional affine subpaces, and in [15] for more algebraic affine surfaces.

The main goal of this paper is to develop a Fourier analytic approach to the study of incidence problems for a reasonably general class of families of manifolds of codimension 1. Our results depend on certain regularity bounds of operators which average functions over families of smooth curves and surfaces in Euclidean space. The properties of such operators have been studied for many years in the context of harmonic analysis and PDEs. See, for example, 27] and the references therein for a thorough description of the subject area. We shall see that $L^{2}$-Sobolev bounds for averaging operators can be converted to incidence theorems for corresponding surfaces and "homogeneous" point sets. We will also give specific examples of curves and surfaces to which our incidence bounds apply. The main requirement is that the said regularity-improving bounds should hold, and that has been proved by harmonic analysts in a wide variety of settings, typically under assumptions involving smoothness and curvature. (By contrast, combinatorial methods tend to ignore properties of this type altogether, relying instead on geometric assumptions similar to those stated above for pseudolines.) The homogeneity assumption on the point set, formulated rigorously in Definition 2.1. means that the point set is uniformly distributed throughout a fixed cube and eliminates the lower-dimensional obstructions described earlier. This condition originated in analysis literature and was used in the context of incidence bounds, e.g., in [25, [24, [15]. 
We mention that another interesting type of geometric obstructions occurs in counting incidences between point sets and manifolds of higher codimension. For example, the Szemerédi-Trotter bound (1.1) extends trivially to point-line arrangements in higher dimensions, as can be seen by projecting the arrangement on a fixed plane and observing that each incidence in the original arrangement corresponds to an incidence in the projected one. However, the converse of the last statement is false, and one expects that the number of incidences in a truly higher-dimensional arrangement should be substantially lower. This is indeed confirmed, e.g., in [25]. The challenge is thus to find a good notion of a "higher-dimensional" arrangement under which sharp bounds, or at least bounds better than those in two dimensions, can be obtained. See, for example, [2, [15, 21, 20] for the case of incidences between points and lines or curves in higher dimensions. Connections between this type of problem and certain questions in analysis is described and thoroughly referenced in [30; see also [19], which puts some recent work in harmonic analysis involving combinatorics of circles and spheres in a broader perspective. We do not address this class of problems here, but we do set up a framework which we hope to apply to such questions in the future.

\section{The Main Result}

Our results concern upper bounds on the number of incidences between "homogeneous" point sets (see below) and families of surfaces for which certain analytic bounds are known to hold. A model case that the reader should keep in mind is that of a finite and homogeneous point set $A \subset[0,1]^{d}$ and the family of spheres $\left\{S_{a}\right\}_{a \in A}$, where $S_{a}=\left\{x \in \mathbb{R}^{d}:|x-a|=.1\right\}$. We will obtain an upper bound on the number of pairs $\left\{\left(a, a^{\prime}\right) \in A \times A: a^{\prime} \in S_{a}\right\}$ (equivalently, $\left\{\left(a, a^{\prime}\right) \in A \times A:\left|a-a^{\prime}\right|=.1\right\}$ ). While this particular case has already been investigated by combinatorial methods and we are not able to improve the known bounds here, our results apply to other classes of surfaces as well, for example spheres with varying radii (depending on $a$ ) or translates of smooth convex hypersurfaces satisfying appropriate analytic conditions. We do not know of any combinatorial methods that would work in the latter case; simple extensions of the known methods are not sufficient, basically because it is difficult to control intersections of convex bodies in dimensions 3 and higher. There is some overlap between the results presented here and those of Laba and Solymosi [15], where combinatorial assumptions on the surfaces are made instead of analytic assumptions.

We first describe the point sets that we work with.

Definition 2.1. Let $C_{0}, c_{0}$ be positive constants with $0<c_{0}<C_{0}$. We say that a set $A \subset[0,1]^{d}, d \geq 2$, is $\left(C_{0}, c_{0}, k_{0}\right)$-homogeneous if every cube of sidelength $c_{0}|A|^{-1 / d}$ contains at most $k_{0}$ points of $A$, and if every cube of sidelength $C_{0}|A|^{-1 / d}$ contains at least one point of $A$.

We recall that an infinite point set $A \subset \mathbb{R}^{d}$ is called a Delone (a.k.a. Delauney or well-distributed) set if there exist $0<c_{A}<C_{A}$ such that every cube of sidelength $c_{A}$ contains at most $k_{0}$ points of $A$, and every cube of sidelength $C_{A}$ contains at least one point of $A$. Thus the rescaled and truncated sets,

$$
A_{t}=[0,1]^{d} \cap\{t a: a \in A\},
$$


are $\left(C_{A}, c_{A}, k_{0}\right)$-homogeneous, with the same constants for all $t>1$. Delone sets have been studied recently in connection with higher-dimensional incidence theorems and with the Erdős and Falconer distance problems; see, e.g., 11, [12, 25, [10], 24], [13].

The Fourier transform of a Schwartz class function $f$ is defined by the formula

$$
\hat{f}(\xi)=\int_{\mathbb{R}^{d}} e^{-2 \pi i x \cdot \xi} f(x) d x .
$$

In what follows, the homogeneous Sobolev space $L_{\gamma}^{2}\left(\mathbb{R}^{d}\right)$ is the closure of the Schwartz class $\mathcal{S}\left(\mathbb{R}^{d}\right)$ in the norm

$$
\|u\|_{L_{\gamma}^{2}\left(\mathbb{R}^{d}\right)}^{2}=\int_{\mathbb{R}^{d}}|\xi|^{2 \gamma}|\widehat{u}(\xi)|^{2} d \xi .
$$

Definition 2.2. Let $\mathcal{G}=\left\{\Gamma_{x}\right\}_{x \in(0,1)^{d}}$ be a continuous family of smooth manifolds in $\mathbb{R}^{d}$.

(i) We say that $\mathcal{G}$ is regular if there is a smooth function $\Phi: \mathbb{R}_{x, y}^{2 d} \rightarrow \mathbb{R}$ such that $\Gamma_{x}=\{y: \Phi(x, y)=0\}$ for all $x \in[0,1]^{d}$ and that $\left|\nabla_{x} \Phi(x, y)\right|>\epsilon_{0}$, $\left|\nabla_{y} \Phi(x, y)\right|>\epsilon_{0}$ for some $\epsilon_{0}>0$ and all $(x, y)$ with $\Phi(x, y)=0$.

(ii) We say that $\mathcal{G}$ is $\gamma$-regular if the averaging operator $T$, given by

$$
T f(x)=\int_{\Gamma_{x}} f(y) d \sigma_{x}(y),
$$

where $\sigma_{x}$ is the Lebesgue measure 1 on $\Gamma_{x}$, obeys the following Sobolev estimate:

$$
\|T f\|_{L_{\gamma}^{2}\left(\mathbb{R}^{d}\right)} \lesssim\|f\|_{L^{2}\left(\mathbb{R}^{d}\right)} .
$$

(iii) We say that $\mathcal{G}$ is strongly $\gamma$-regular if the following holds for all $t \in$ $\left(-c_{R}, c_{R}\right)$, where $c_{R}$ is a small fixed positive constant. Let $\Gamma_{x, t}=\{y$ : $\Phi(x, y)=t\}$. Then the families $\mathcal{G}_{t}=\left\{\Gamma_{x, t}\right\}_{x \in(0,1)^{d}}$ are $\gamma$-regular, with the constant uniform for all $t \in\left(-c_{R}, c_{R}\right)$.

We remark that, since $L^{p}$ functions are defined only up to sets of measure zero, the restriction of such a function to a lower-dimensional submanifold need not always be defined or measurable. Nonetheless, under certain natural conditions (examples of which will be given shortly), $T f(x)$ is defined for almost all $x$ and obeys (2.1). In fact, for compact surfaces, (2.1) with $\gamma=0$ trivially holds in our setup by the Minkowski integral inequality. Plugging this into our numerology would result in a trivial version of Theorem 2.3 with incidence bound $N^{2-\frac{1}{d}}$, reflecting the observation that each $\Gamma_{x}$ can be incident to at most $N^{(d-1) / d}$ points due to dimensionality considerations and the homogeneity assumption. It is easy to construct counterexamples showing that the estimate (2.1) generally cannot hold with $\gamma>\frac{d-1}{2}$; see, e.g., 9 .

We now state our main results.

Theorem 2.3. Let $A$ be a $\left(C_{0}, c_{0}, k_{0}\right)$-homogeneous set in $\mathbb{R}^{d}, d \geq 2$, with $|A|=N$. Suppose that the family $\mathcal{G}=\left\{\Gamma_{x}\right\}_{x \in(0,1)^{d}}$ is strongly $\gamma$-regular for some $\gamma>0$. Let $s \in(d-\gamma, d)$. Then for all $\delta \leq c N^{-1 / s}$, where $c$ is small enough,

$$
\left|\left\{\left(a, a^{\prime}\right) \in A \times A: a^{\prime} \in \Gamma_{a}^{\delta}\right\}\right| \lesssim N^{2-\frac{1}{s}} .
$$

\footnotetext{
${ }^{1}$ We do not normalize $\sigma_{x}$, so that the measure of $\Gamma_{x}$ is equal to $\lim \inf _{\delta \rightarrow 0}\left|\Gamma_{x}^{\delta}\right|$.
} 
In particular, we have the incidence bound

$$
\left|\left\{\left(a, a^{\prime}\right) \in A \times A: a^{\prime} \in \Gamma_{a}\right\}\right| \lesssim N^{2-\frac{1}{d-\gamma}} .
$$

Here and throughout the rest of the paper, the implicit constants in the $\lesssim$ and $\lesssim$ symbols depend on the constants $c_{0}, C_{0}$ in Definition 2.1 the constants $C_{L}, c_{R}, \epsilon_{0}$ in Definition 2.2. and the exponent $s$. However, they are always independent of $N$.

Note that if $A$ and $B$ are two $\left(C_{0}, c_{0}, k_{0}\right)$-homogeneous sets, then $A \cup B$ is $\left(C_{0}, c_{0}, 2 k_{0}\right)$-homogeneous. Therefore our results apply just as well (possibly with different constants) to quantities of the form

$$
\left|\left\{(a, b) \in A \times B: b \in \Gamma_{a}\right\}\right|,
$$

where $A, B$ are two different $\left(C_{0}, c_{0}, k_{0}\right)$-homogeneous sets. This comment applies to Theorem 2.3 as well as to all corollaries in the sequel.

\section{Applications}

In this section, we discuss applications of the abstract Theorem 2.3 to specific incidence problems. We continue to assume that $A \subset[0,1]^{d}$ is a $\left(C_{0}, c_{0}, k_{0}\right)$ homogeneous set with $|A|=N$.

We first discuss a group of results obtained by combining Theorem 2.3 and the classical theory of Fourier integral operators. We need a definition.

Definition 3.1. Suppose that $\mathcal{G}=\left\{\Gamma_{x}\right\}_{x \in \mathbb{R}^{d}}$ is a family of $(d-1)$-dimensional hypersurfaces in $\mathbb{R}^{d}$ defined by $\Gamma_{x}=\{y: \Phi(x, y)=0\}$, where $\Phi: \mathbb{R}^{d} \times \mathbb{R}^{d} \rightarrow \mathbb{R}$ is a smooth function. We will say that $\mathcal{G}$ satisfies the rotational curvature condition of Phong and Stein (see [18] and [22], Theorem 6.2.1 and Corollary 6.2.3) if the Monge-Ampère determinant

$$
\mathcal{M}(\Phi)=\left(\begin{array}{cccc}
0 & \frac{\partial \Phi}{\partial x_{1}} & \cdots & \frac{\partial \Phi}{\partial x_{d}} \\
\frac{\partial \Phi}{\partial y_{1}} & \frac{\partial^{2} \Phi}{\partial x_{1} \partial y_{1}} & \ldots & \frac{\partial^{2} \Phi}{\partial x_{d} \partial y_{1}} \\
\vdots & \vdots & \ddots & \vdots \\
\frac{\partial \Phi}{\partial y_{d}} & \frac{\partial^{2} \Phi}{\partial x_{1} \partial y_{d}} & \cdots & \frac{\partial^{2} \Phi}{\partial x_{d} \partial y_{d}}
\end{array}\right)
$$

restricted to the set where $\Phi(x, y)=0$, does not vanish.

Corollary 3.2. Suppose that $\mathcal{G}=\left\{\Gamma_{x}\right\}_{x \in \mathbb{R}^{d}}$, where $\Gamma_{x}=\{y: \Phi(x, y)=0\}$, satisfies the Phong-Stein condition (3.1) in Definition 3.1. Let $s \in\left(\frac{d+1}{2}, d\right)$. Then for any $\delta<c N^{-1 / s}$, with c small enough,

$$
\left|\left\{\left(a, a^{\prime}\right) \in A \times A: a^{\prime} \in \Gamma_{a}^{\delta}\right\}\right| \lesssim N^{2-\frac{1}{s}}
$$

In particular,

$$
\left|\left\{\left(a, a^{\prime}\right) \in A \times A: a^{\prime} \in \Gamma_{a}\right\}\right| \lesssim N^{\frac{2 d}{d+1}} .
$$

Corollary 3.2 follows from Theorem 2.3 in view of the main result of [18] which says that under the assumptions of Corollary 3.2, the estimate (2.1) holds with $\gamma=\frac{d-1}{2}$. Note also that the Phong-Stein condition implies that $\left|\nabla_{x} \Phi\right|$ and $\left|\nabla_{y} \Phi\right|$ are bounded from below away from 0 on $\Gamma_{x}$. The constants hidden in the $\lesssim$ and $\lesssim$ symbols will clearly depend on the function $\Phi$.

While the Monge-Ampère condition in Corollary 3.2 is often interpreted as a curvature condition, it also allows for certain families of "flat" hypersurfaces. For example, it is satisfied by the family of hyperplanes $\Gamma_{x}=\left\{y \in(0,1)^{d}: x \cdot y=1\right\}$. 
The corresponding estimate in Corollary 3.2 yields a special case of the SzemerédiTrotter theorem in two dimensions and a non-trivial incidence bound in higher dimensions.

As a special case of Corollary 3.2 we obtain the following.

Corollary 3.3. Let $d \geq 2$, and let $r(x)$ be a smooth function $[0,1]^{d} \rightarrow(0, \infty)$ such that $|\nabla r(x)| \leq c<1$. Let $\Gamma_{x}=\{y:|x-y|=r(x)\}$. Then the conclusions of Corollary 3.2 hold; in particular, we have

$$
\left|\left\{\left(a, a^{\prime}\right) \in A \times A:\left|a-a^{\prime}\right|=r(a)\right\}\right| \lesssim N^{\frac{2 d}{d+1}} .
$$

To prove the corollary, it suffices to verify that the Phong-Stein condition holds for $\Phi(x, y)=|x-y|^{2}-r(x)^{2}$. We do this in Section 6 .

If $d=2$ and $r(x) \equiv r_{0}$ is fixed, Corollary 3.3 says in particular that the number of pairs $a, a^{\prime} \in A$ such that $\left|a-a^{\prime}\right|=r_{0}$ (i.e., the number of incidences between the $N$ points of $A$ and $N$ circles of radius $r_{0}$ and centered at those points) is $\lesssim N^{4 / 3}$. This is a partial result on the "unit distance" conjecture of Erdős, which asserts that if $P \subset \mathbb{R}^{2}$ is a set of cardinality $N$ and $r_{0}>0$ is fixed, then

$$
\#\left\{(a, b) \in P \times P:|a-b|=r_{0}\right\} \lesssim N \sqrt{\log (N)} .
$$

The best-known partial result to date follows from the Szemerédi-Trotter theorem and yields $N^{4 / 3}$ on the right side of (3.5) [26]. Our result matches this up to the endpoint. On the one hand, our point set is somewhat special and we do lose the endpoint. On the other hand, our theorem applies also to circles $\{y:|a-y|=r(a)\}$ of varying radii, as well as to thin annuli $\{y: r(a) \leq|a-y| \leq r(a)+\delta\}$ with $\delta=$ $c N^{-2 / 3}$. These extensions do not appear to follow from the known combinatorial theorems (Szemerédi-Trotter, Pach-Sharir) in any straightforward way.

In three dimensions, the best known estimate on the number of unit distances is $O\left(N^{\frac{3}{2}+\epsilon}\right)$ for any $\epsilon>0$ [5. Our result matches this estimate in the case of homogeneous sets, except that [5] gives an explicit form of the endpoint $o\left(N^{\epsilon}\right)$ factor. (The best-known lower bound is $N^{4 / 3} \log \log N$.) In dimensions $d \geq 4$, the number of unit distances in a general point set of cardinality $N$ can be of the order $N^{2}$, as demonstrated by the example in Section 1. Nonetheless, Corollary 3.3 still yields a non-trivial bound for homogeneous sets in higher dimensions.

We note that the condition $|\nabla r(x)|<c<1$ has an appealing geometrical interpretation: it is a slightly strengthened quantitative version of the statement that that no sphere $\Gamma_{x}$ is entirely contained within another sphere $\Gamma_{y}$.

We further note that our theorem yields the same conclusions for the unit distance problem if the Euclidean norm is replaced by a non-isotropic norm in which the unit sphere is a convex set with sufficiently smooth boundary and everywhere non-vanishing curvature. It is well known that combinatorial methods run into difficulties for this type of problem, especially in higher dimensions. The known incidence bounds for spheres use very specific geometric information, for example that two spheres always intersect along a circle; on the other hand, the intersection curves of more general convex surfaces can be almost impossible to control. The related paper [15] gives an incidence bound for algebraic surfaces in $\mathbb{R}^{3}$, with exponent depending on the algebraic degree of the surfaces. The bounds in Corollaries 3.2, 3.3 improve on that of [15] for algebraic surfaces of high enough degree.

In Corollary 3.3. we assumed that the radius $r(x)$ of the sphere centered at $x$ obeyed $|\nabla r(x)|<c<1$. If we assume instead that $|\nabla r(x)|>C>1$ for all $x$, then 
it turns out that a weaker estimate can be proved in dimensions $d \geq 3$. Again, our estimate is in fact more general and applies just as well to translated and dilated copies of a fixed curved hypersurface. We first give the analytic statement of the result.

Corollary 3.4. Let $d \geq 3$. Let $\mathcal{G}=\left\{\Gamma_{x}\right\}_{x \in[0,1]^{d}}$, where $\Gamma_{x}=\{y: \Phi(x, y)=0\}$ and $\Phi: \mathbb{R}^{d} \times \mathbb{R}^{d} \rightarrow \mathbb{R}$ is a smooth function. Assume that:

(i) $\left|\nabla_{x} \Phi(x, y)\right|>\epsilon_{0},\left|\nabla_{y} \Phi(x, y)\right|>\epsilon_{0}$ for some $\epsilon_{0}>0$ and all $(x, y)$ with $\Phi(x, y)=0$,

(ii) there is a smooth function $r(x)$ with values in $[a, b]$ for some $0<a<b<\infty$ such that $\Phi(x, y)=f\left(\frac{y-x}{r(x)}\right)$ for some smooth function $f: \mathbb{R}^{d} \rightarrow \mathbb{R}$, so that $\Gamma_{x}=$ $x+r(x) \Gamma$ for a fixed $\Gamma:=\Gamma_{0}$,

(iii) $\Gamma$ (defined above) is a smooth closed hypersurface with everywhere nonvanishing Gaussian curvature.

Let $s \in\left(\frac{d+2}{2}, d\right)$. Then for any $\delta<c N^{-1 / s}$, with c small enough,

$$
\left|\left\{\left(a, a^{\prime}\right) \in A \times A: a^{\prime} \in \Gamma_{a}^{\delta}\right\}\right| \lesssim N^{2-\frac{1}{s}} .
$$

In particular,

$$
\left|\left\{\left(a, a^{\prime}\right) \in A \times A: a^{\prime} \in \Gamma_{a}\right\}\right| \lesssim N^{\frac{2 d+2}{d+2}} .
$$

We now give a variant of the above corollary which is slightly weaker, but easier to apply to combinatorial problems where the defining function $\Phi$ is not given explicitly.

Corollary 3.5. Let $d \geq 3$. Let $K$ be a convex body in $\mathbb{R}^{d}$ whose interior contains 0 and such that $\Gamma=\partial K$ is a smooth closed hypersurface with everywhere nonvanishing Gaussian curvature. Let $\Gamma_{x}=x+r(x) \Gamma$, where $r(x)$ is a smooth function with values in $[a, b]$ for some $0<a<b<\infty$. Assume that either

$$
|\nabla r(x)|>C_{0}>M \text { for all } x \in[0,1]^{d}
$$

or

$$
|\nabla r(x)|<c_{0}<m \text { for all } x \in[0,1]^{d},
$$

where $m=\min _{x \in \Gamma}|x|$ and $M=\max _{x \in \Gamma}|x|$. Let $s \in\left(\frac{d+2}{2}, d\right)$. Then for any $\delta<c N^{-1 / s}$, where $c$ is small enough, the estimates (3.6) and (3.7) hold.

In particular, the assumptions (therefore the conclusion) of the corollary hold in the special case when $\Gamma_{x}=\{y:|x-y|=r(x)\}$ and $r(x)$ is a smooth function with $|\nabla r(x)|>C_{0}>1$ for all $x$, since then $\Gamma$ is the unit sphere and $m=M=1$. (The case $|\nabla r(x)|<c_{0}<1$ is already covered by Corollary 3.3)

If $d=2$, the allowed range of $s$ in the first estimate (3.6) is empty. In this case, the estimate (3.7) is the trivial incidence bound, which follows from the dimensionality of $\Gamma_{x}$ and the homogeneity of $A$.

\section{Proof of Theorem 2.3}

The proof is based on a conversion mechanism, first developed in [11, 12] in the context of the Falconer distance set problem. Let $A$ and $\left\{\Gamma_{x}\right\}_{x \in(0,1)^{d}}$ be as in the statement of the theorem. Also let $\delta=c N^{-1 / s}$, where $c>0$ is small enough and

$$
d-\gamma<s<d \text {. }
$$


We will prove that

$$
\left|\left\{\left(a, a^{\prime}\right) \in A \times A: a^{\prime} \in \Gamma_{a}^{\delta}\right\}\right| \lesssim N^{2-\frac{1}{s}} .
$$

This clearly implies the theorem, since the inequality clearly remains valid if the $\delta$ on the left side of (4.2) is replaced by a smaller number.

We define

$$
f(x)=N^{-1} \delta^{-d} \sum_{a \in A} \phi\left(\frac{x-a}{\delta}\right),
$$

where $\phi: \mathbb{R}^{d} \rightarrow[0, \infty)$ is a smooth function such that $\phi(x) \equiv 1$ for $|x| \leq 1$ and $\phi(x) \equiv 0$ for $|x| \geq 2$. Then $f$ is supported on a $\delta$-neighbourhood of $[0,1]^{d}$ and $\int_{\mathbb{R}^{d}} f \approx 1$. Let $d \mu=f d x$. We also let

$$
E=\bigcup_{a \in A}\{x:|x-a| \leq \delta\} .
$$

For each pair $\left(a, a^{\prime}\right) \in A \times A$, let

$$
B_{a, a^{\prime}}:=\left\{(x, y):|x-a| \leq c \delta,\left|y-a^{\prime}\right| \leq c \delta\right\} .
$$

We will assume that the constant $c<1$ is small enough so that the sets $B_{a, a^{\prime}}$ are pairwise disjoint. We also have $B_{a, a^{\prime}} \subset E \times E$.

We further observe that if $c$ is sufficiently small (which we will assume henceforth), and if $(x, y) \in B_{a, a^{\prime}}$ and $a^{\prime} \in \Gamma_{a}^{c \delta}$, then $y \in \Gamma_{x}^{\delta}$. This follows from the uniform continuity of the defining function $\Phi$. Hence, with $c$ as above,

$$
N^{-2}\left|\left\{\left(a, a^{\prime}\right) \in A \times A: a^{\prime} \in \Gamma_{a}^{c \delta}\right\}\right| \lesssim \mu \times \mu\left\{(x, y) \in E \times E: y \in \Gamma_{x}^{\delta}\right\} .
$$

Let

$$
\begin{aligned}
& T^{\delta} g(x)=\int_{\Gamma_{x}^{\delta}} g(y) d y, \\
& T_{t} g(x)=\int_{\Gamma_{x, t}} g(y) d \sigma_{x, t}(y),
\end{aligned}
$$

where $\Gamma_{x, t}$ are as in Definition 2.2 and $d \sigma_{x, t}$ is the surface measure on $\Gamma_{x, t}$. Using a change of variables, and invoking the regularity of $\Phi$ again, we estimate

$$
\begin{aligned}
\mu \times \mu\left\{(x, y) \in E \times E: y \in \Gamma_{x}^{\delta}\right\} & =\left\langle T^{\delta} f(x), f(x)\right\rangle \\
& \lesssim \int_{0}^{C \delta}\left\langle T_{t} f(x), f(x)\right\rangle d t .
\end{aligned}
$$

We now use (2.1) to bound the last integrand uniformly in $t$.

Fix Schwartz class functions $\eta_{0}(\xi)$ supported in $|\xi| \leq 4$ and $\eta(\xi)$ supported in the spherical shell $1<|\xi|<4$ such that the quantities $\eta_{0}(\xi), \eta_{j}(\xi)=\eta\left(2^{-j} \xi\right), j \geq 1$ form a partition of unity.

Write $f=\sum_{j=0}^{\infty} f_{j}$, where $\widehat{f}_{j}(\xi)=\widehat{f}(\xi) \eta_{j}(\xi)$. Then

$$
\left\langle T_{t} f, f\right\rangle=\sum_{j, k}\left\langle T_{t} f_{j}, f_{k}\right\rangle=\sum_{|j-k| \leq K}+\sum_{|j-k|>K},
$$

where $K$ is a large enough constant. We will estimate the two sums separately, starting with the second one. Note that this is very easy in the translation-invariant 
case when $\Gamma_{x}=\Gamma+x$ for a fixed $\Gamma$ (i.e., $\Phi(x, y)$ depends only on $x-y$ ), since then, by Plancherel's theorem,

$$
\begin{gathered}
\left\langle T_{t} f_{j}, f_{k}\right\rangle=\left\langle\widehat{T_{t} f_{j}}, \widehat{f_{k}}\right\rangle=\left\langle\widehat{f_{j} * \sigma_{0, t}}, \widehat{f_{k}}\right\rangle \\
=\left\langle\widehat{f_{j}} \widehat{\sigma_{0, t}}, \widehat{f_{k}}\right\rangle=0 \text { if }|j-k|>1
\end{gathered}
$$

and the second sum vanishes if we let $K=1$. In the general case, we need the following lemma.

Lemma 4.1. Assume that $K>0$ is large enough. Then for any $M$ there exists $C_{M}>0$ such that for all $k, j$ with $|j-k|>K$,

$$
\left\langle T f_{j}, f_{k}\right\rangle \leq C_{M} 2^{-M \max (j, k)} .
$$

We defer the proof of the lemma until the next section. Applying the lemma with $M=1$, we estimate the second sum in (4.7):

$$
\sum_{|j-k|>K}\left|\left\langle T_{t} f_{j}, f_{k}\right\rangle\right| \lesssim \sum_{j=0}^{\infty} \sum_{k=j+K+1}^{\infty} 2^{-k}+\sum_{k=0}^{\infty} \sum_{j=k+K+1}^{\infty} 2^{-j} \lesssim 1 .
$$

Turning to the first sum in (4.7), we write

$$
\sum_{|j-k| \leq K}\left|\left\langle T_{t} f_{j}, f_{k}\right\rangle\right|=\sum_{r=-K}^{K} \sum_{j=0}^{\infty}\left|\left\langle T_{t} f_{j}, f_{j+r}\right\rangle\right|
$$

where we put $f_{k} \equiv 0$ for $k<0$. By (2.1) and the Cauchy-Schwarz inequality we have

$$
\left|\left\langle T_{t} f_{j}, f_{j+r}\right\rangle\right| \leq\left\|T_{t} f_{j}\right\|_{2}\left\|f_{j+r}\right\|_{2} \leq 2^{-j \gamma}\left\|f_{j}\right\|_{2}\left\|f_{j+r}\right\|_{2} .
$$

We claim that

$$
\left\|f_{j}\right\|_{2}^{2} \lesssim 2^{j(d-s)}
$$

Assuming (4.10) for the moment, we conclude that

$$
\sum_{|j-k| \leq K}\left|\left\langle T_{t} f_{j}, f_{k}\right\rangle\right| \lesssim \sum_{r=-K}^{K} \sum_{j=0}^{\infty} 2^{-j \gamma} 2^{j(d-s) / 2} 2^{(j+r)(d-s) / 2} \lesssim \sum_{j} 2^{-j \gamma+j(d-s)} \lesssim 1,
$$

provided that (4.1) holds. Combining (4.8) and (4.11), we see that

$$
\left|\left\langle T_{t} f, f\right\rangle\right| \lesssim 1 .
$$

Finally, we plug this into (4.6) and get

$$
\mu \times \mu\left\{(x, y) \in E \times E: y \in \Gamma_{x}^{\delta}\right\} \lesssim \delta \lesssim N^{-1 / s} .
$$

The conclusion (4.2) follows from this and (4.4).

It remains to prove (4.10). By the Fourier support localization of $f_{j}$ and Plancherel's theorem, we have

$$
\left\|f_{j}\right\|_{2}^{2}=\left\|\widehat{f}_{j}\right\|_{2}^{2} \lesssim 2^{j(d-s)} \int|\widehat{f}(\xi)|^{2}|\xi|^{-d+s} d \xi .
$$

On the other hand, the integral on the right-hand side is the $s$-energy of $\mu$, i.e.

$$
\int|\widehat{f}(\xi)|^{2}|\xi|^{-d+s} d \xi=c_{d, s} \iint|x-y|^{-s} d \mu(x) d \mu(y):=I_{s},
$$


where $c_{d, s}$ is an explicit constant depending only on $d$ and $s$ (see 31]). Thus it suffices to prove that

$$
I_{s} \lesssim 1
$$

Indeed, we write

$$
\begin{gathered}
I_{s}=N^{-2} \delta^{-2 d} \sum_{a, a^{\prime} \in A} \iint|x-y|^{-s} \phi\left(\frac{x-a}{\delta}\right) \phi\left(\frac{y-a^{\prime}}{\delta}\right) \\
=N^{-2} \delta^{-2 d}\left(\sum_{a, a^{\prime} \in A:\left|a-a^{\prime}\right| \leq 4 \delta}+\sum_{j=2}^{\infty} \sum_{a, a^{\prime} \in A: 2^{j} \delta<\left|a-a^{\prime}\right| \leq 2^{j+1} \delta}\right):=N^{-2} \delta^{-2 d}\left(S_{1}+S_{2}\right) .
\end{gathered}
$$

We start with the first term. If $\delta \lesssim N^{-1 / s}$ with $0<s<d$, then for large $N$ we can only have $\left|a-a^{\prime}\right|<4 \delta, a, a^{\prime} \in A$, if $a=a^{\prime}$. Hence

$$
\begin{aligned}
S_{1}=\sum_{a} \iint|x-y|^{-s} \phi\left(\frac{x-a}{\delta}\right) \phi\left(\frac{y-a}{\delta}\right) & \lesssim N \iint|u|^{-s} \phi\left(\frac{u+y}{\delta}\right) \phi\left(\frac{u}{\delta}\right) \\
& \lesssim N \delta^{d} \int_{|u| \leq 2 \delta} \int|u|^{-s} d s \lesssim N \delta^{2 d-s} .
\end{aligned}
$$

In the second term, if $2^{j} \delta<\left|a-a^{\prime}\right| \leq 2^{j+1} \delta$, then $2^{j-1} \delta<|x-y| \leq 2^{j+2} \delta$ on the support of the integrand. For each $a \in A$, there are about $N \cdot\left(2^{j} \delta\right)^{d}$ points $a^{\prime} \in A$ with $2^{j} \delta<\left|a-a^{\prime}\right| \leq 2^{j+1} \delta$. Hence

$$
\begin{aligned}
S_{2} & \lesssim \sum_{j=2}^{\infty} N^{2}\left(2^{j} \delta\right)^{d}\left(2^{j} \delta\right)^{-s} \delta^{2 d} \\
& \lesssim \sum_{j=2}^{\infty} N^{2} \delta^{3 d-s} 2^{j(d-s)} \lesssim N^{2} \delta^{3 d-s},
\end{aligned}
$$

where we again used that $0<s<d$. Combining the estimates on $S_{1}$ and $S_{2}$, we get

$$
I_{s} \lesssim N^{-2} \delta^{-2 d}\left(N \delta^{2 d-s}+N^{2} \delta^{3 d-s}\right)=N^{-1} \delta^{-s}+\delta^{d-s} \lesssim 1,
$$

as claimed. This proves (4.10) and completes the proof of Theorem 2.3

\section{Proof of Lemma 4.1}

To simplify the notation, we will only prove the lemma with $T_{t}$ replaced by the operator $T$ as in Definition 2.2. It will be clear from the proof that the same estimates hold for $T_{t}$ for $|t| \leq C \delta$, with constants uniform in $t$.

We write

$$
T f(x)=\int_{\{y: \Phi(x, y)=0\}} f(y) \psi(x, y) d \sigma_{x}(y),
$$

where $\psi$ is smooth and compactly supported and $d \sigma_{x}(y)$ is the surface measure on $\Gamma_{x}=\{y: \Phi(x, y)=0\}$. Using the $\gamma$-regularity assumption, and shrinking the support of $\psi$ if necessary, we may assume that $C^{-1} \leq\left|\nabla_{y} \Phi(x, y)\right| \leq C$ for some $C>0$ and all $x, y \in \operatorname{supp} \psi$. By the definition of Lebesgue measure, we can approximate $T$ by $T_{n}$ as $n \rightarrow \infty$ :

$$
T_{n} f(x)=n \int f(y) \psi(x, y) \psi_{0}(n \Phi(x, y)) d y
$$


where $\psi_{0}$ is a smooth cutoff function supported in $[-1,1]$. Hence it suffices to prove our estimate for $T_{n}$ with constants uniform in $n$. Applying Fourier inversion twice, to $\psi_{0}$ and then to $f$, we write

$$
\begin{aligned}
T_{n} f(x) & =\iint e^{2 \pi i t \Phi(x, y)} f(y) \psi(x, y) \widehat{\psi}_{0}\left(n^{-1} t\right) d t d y \\
& =\iiint e^{2 \pi i \mu \cdot y} e^{2 \pi i t \Phi(x, y)} \widehat{f}(\mu) \psi(x, y) \widehat{\psi}_{0}\left(n^{-1} t\right) d t d y d \mu .
\end{aligned}
$$

It follows that

$$
\widehat{T_{n} f}(\xi)=\iiint \int e^{2 \pi i \mu \cdot y} e^{2 \pi i t \Phi(x, y)} \widehat{f}(\mu) \psi(x, y) \widehat{\psi}_{0}\left(n^{-1} t\right) e^{-2 \pi i x \cdot \xi} d t d y d x d \mu .
$$

Using Plancherel's theorem, we write $\left\langle T_{n} f_{j}, f_{k}\right\rangle$ as

$$
\begin{aligned}
& \left\langle T_{n} f_{j}, f_{k}\right\rangle=\left\langle\widehat{T_{n} f_{j}}, \widehat{f}_{k}\right\rangle \\
& =\iiint \iint e^{2 \pi i t \Phi(x, y)} e^{-2 \pi i x \cdot \xi} e^{2 \pi i y \cdot \mu} \widehat{f}_{j}(\mu) \widehat{f}_{k}(\xi) \psi(x, y) \widehat{\psi}_{0}\left(n^{-1} t\right) d t d y d x d \mu d \xi \\
& =\iiint \widehat{f}_{j}(\mu) \widehat{f}_{k}(\xi) I_{j k}(\xi, \mu, t) \widehat{\psi}_{0}\left(n^{-1} t\right),
\end{aligned}
$$

where

$$
\begin{gathered}
I_{j k}(\xi, \mu, t)=\phi_{0}\left(2^{-j}|\mu|\right) \phi_{0}\left(2^{-k}|\xi|\right) I(\xi, \mu, t) \\
I(\xi, \mu, t)=\iint e^{2 \pi i t \Phi(x, y)} e^{-2 \pi i x \cdot \xi} e^{2 \pi i y \cdot \mu} \psi(x, y) d x d y
\end{gathered}
$$

and $\phi_{0}$ is a fixed function in $C_{0}^{\infty}\left(\mathbb{R}^{d}\right)$ equal to 1 on $\{1 \leq|x| \leq 10\}$ and vanishing on $\left\{|x| \leq \frac{1}{2}\right\}$.

We claim that if $|j-k|>K$, where $K$ is a large enough constant, then for any $M$ there is a constant $C_{M}$ such that

$$
\left|I_{j k}(\xi, \mu, t)\right| \leq C_{M} 2^{-M \max (j, k)},
$$

uniformly in $\xi, \mu, t$. Assuming this, the proof of the lemma is completed as follows. By (5.1) and (5.3), we have

$$
\left|\left\langle T_{n} f_{j}, f_{k}\right\rangle\right| \lesssim C_{M} 2^{-M \max (j, k)}\left\|\widehat{f}_{j}\right\|_{1}\left\|\widehat{f}_{k}\right\|_{1}
$$

By the Cauchy-Schwarz inequality and (4.10),

$$
\left\|\widehat{f}_{j}\right\|_{1} \leq\left\|\widehat{f}_{j}\right\|_{2}\left|\operatorname{supp} f_{j}\right|^{1 / 2} \lesssim 2^{j d / 2} 2^{j d / 2} \lesssim 2^{j d}
$$

so that

$$
\left|\left\langle T_{n} f_{j}, f_{k}\right\rangle\right| \lesssim C_{M} 2^{-M \max (j, k)} 2^{j d} 2^{k d} \lesssim C_{M} 2^{-(M-2 d) \max (j, k)} .
$$

Relabelling the constants, we get the conclusion of the lemma.

It remains to prove (5.3). The idea is that $I$ is an oscillatory integral with critical points given by

$$
t \nabla_{x} \Phi(x, y)=\xi ; \quad t \nabla_{y} \Phi(x, y)=-\mu .
$$

If $|j-k| \geq K$, where $K$ is large enough depending on $c_{0}, C_{0}$, then at least one of the cutoff functions $\phi_{0}\left(2^{-j}|\mu|\right), \phi_{0}\left(2^{-k}|\xi|\right)$ is supported away from the critical points; hence we can estimate $I_{j k}$ using the easy part of the stationary phase method which only involves integration by parts. 
The details are as follows. Recall that we are assuming that

$$
c_{0} \leq\left|\nabla_{x} \Phi(x, y)\right| \leq C_{0}, c_{0} \leq\left|\nabla_{y} \Phi(x, y)\right| \leq C_{0},
$$

for some positive constants $c_{0}, C_{0}$. We have

$$
\frac{1}{2 \pi i\left(t \frac{\partial \Phi}{\partial x_{m}}+\xi_{m}\right)} \frac{\partial}{\partial x_{m}} e^{2 \pi i(t \Phi(x, y)+x \cdot \xi)}=e^{2 \pi i(t \Phi(x, y)+x \cdot \xi)},
$$

and, similarly,

$$
\frac{1}{2 \pi i\left(t \frac{\partial \Phi}{\partial y_{m}}-\mu_{m}\right)} \frac{\partial}{\partial y_{m}} e^{2 \pi i(t \Phi(x, y)-y \cdot \mu)}=e^{2 \pi i(t \Phi(x, y)-y \cdot \mu)} .
$$

Using (5.5) in (5.2) and integrating $M$ times by parts in $x_{m}$, we see that for any $M$ there is a constant $C_{M}^{\prime}$ (depending on $\Phi$ and the cutoff functions $\psi, \phi_{0}$ but not on $\xi, \mu, t$ or $j, k)$ such that for $m=1, \ldots, d$,

$$
|I(\xi, \mu, t)| \leq C_{M}^{\prime} \max _{x, y}\left|t \frac{\partial \Phi}{\partial x_{m}}+\xi_{m}\right|^{-M} .
$$

If $|t| \leq\left(2 C_{0}\right)^{-1}|\xi|$, where $C_{0}$ is as in (15.4), then $|t \nabla \Phi| \leq \frac{|\xi|}{2}$; hence there is at least one $m$ such that

$$
\left|t \frac{\partial \Phi}{\partial x_{m}}+\xi_{m}\right| \geq d^{-1}|| t \nabla \Phi|-| \xi|| \geq \max \left(\frac{|\xi|}{2 d}, \frac{|t|}{C_{0} d}\right) .
$$

Similarly, if $|t| \geq 2 c_{0}^{-1}|\xi|$, then $|t \nabla \Phi| \geq 2|\xi|$; hence

$$
\left|t \frac{\partial \Phi}{\partial x_{m}}+\xi_{m}\right| \geq \max \left(\frac{|\xi|}{d}, \frac{c_{0}|t|}{2 d}\right)
$$

for at least one $m$. It follows that in both regions,

$$
\left|I_{j k}(\xi, \mu, t)\right| \leq C_{M}^{\prime \prime}(\max (|t|,|\xi|))^{-M}
$$

Similarly, if either $|t| \leq\left(2 C_{0}\right)^{-1}|\mu|$ or $|t| \geq 2 c_{0}^{-1}|\mu|$, we have for any $M$,

$$
\left|I_{j k}(\xi, \mu, t)\right| \leq C_{M}^{\prime \prime}(\max (|t|,|\mu|))^{-M} .
$$

Thus one of (5.9), (15.10) must hold unless we have both $\left(2 C_{0}\right)^{-1}|\xi| \leq|t| \leq 2 c_{0}^{-1}|\xi|$ and $\left(2 C_{0}\right)^{-1}|\mu| \leq|t| \leq 2 c_{0}^{-1}|\mu|$. But then

$$
\frac{1}{4} c_{0} C_{0}^{-1}|\mu| \leq|\xi| \leq 4 c_{0}^{-1} C_{0}|\mu| .
$$

Choose $K$ large enough so that $K \geq 100 c_{0}^{-1} C_{0}$. Then (5.11) fails on the support of $\phi_{0}\left(2^{-j}|\mu|\right) \phi_{0}\left(2^{-k}|\xi|\right)$ whenever $|j-k| \geq K$, so that at least one of (5.9), (5.10) hold. Suppose now that $j-k>K$, so that $|\xi| \leq|\mu|$ on $\operatorname{supp} I_{j k}$. If (5.10) holds, (5.3) follows immediately. If on the other hand (5.10) fails, we must in particular have $|t| \geq\left(2 C_{0}\right)^{-1}|\mu|$. Plugging this into (5.9), we get (5.3) again. 


\section{Proof of Corollary 3.2}

It suffices to show that $\mathcal{M}(\Phi)$, where

$$
\Phi(x, y)=\left(x_{1}-y_{1}\right)^{2}+\cdots+\left(x_{d}-y_{d}\right)^{2}-r(x)^{2},
$$

has determinant bounded away from zero on the set $\{(x, y): \Phi(x, y)=0\}$. We abbreviate $r_{j}=\frac{\partial r}{\partial x_{j}}$. Then

$$
\begin{aligned}
\operatorname{det}[\mathcal{M}(\Phi)] & =\left|\begin{array}{ccccc}
0 & 2\left(y_{1}-x_{1}\right) & 2\left(y_{2}-x_{2}\right) & \ldots & 2\left(y_{d}-x_{d}\right) \\
2\left(x_{1}-y_{1}\right)-2 r r_{1} & -2 & 0 & \ldots & 0 \\
2\left(x_{2}-y_{2}\right)-2 r r_{2} & 0 & -2 & \ldots & 0 \\
\vdots & \vdots & \vdots & \ddots & \vdots \\
2\left(x_{d}-y_{d}\right)-2 r r_{d} & 0 & 0 & \ldots & -2
\end{array}\right| \\
& =(-1)^{d} 2^{d+1} D_{d},
\end{aligned}
$$

where

$$
D_{d}=\left|\begin{array}{ccccc}
0 & x_{1}-y_{1} & x_{2}-y_{2} & \ldots & x_{d}-y_{d} \\
x_{1}-y_{1}-r r_{1} & 1 & 0 & \ldots & 0 \\
x_{2}-y_{2}-r r_{2} & 0 & 1 & \ldots & 0 \\
\vdots & \vdots & \vdots & \ddots & \vdots \\
x_{d}-y_{d}-r r_{d} & 0 & 0 & \ldots & 1
\end{array}\right|
$$

Expanding in the last row, we get

$$
D_{d}=(-1)^{d}\left(x_{d}-y_{d}-r r_{d}\right)\left|\begin{array}{ccccc}
x_{1}-y_{1} & x_{2}-y_{2} & \ldots & x_{d-1}-y_{d-1} & x_{d}-y_{d} \\
1 & 0 & \ldots & 0 & 0 \\
0 & 1 & \ldots & 0 & 0 \\
\vdots & \vdots & \ddots & \vdots & \vdots \\
0 & 0 & \ldots & 1 & 0
\end{array}\right|+D_{d-1} .
$$

Expanding the remaining determinant in the last column yields

$$
D_{d}=-\left(x_{d}-y_{d}\right)^{2}+r r_{d}\left(x_{d}-y_{d}\right)+D_{d-1} .
$$

It follows by induction that

$$
D_{d}=-|x-y|^{2}+r(x)(x-y) \cdot \nabla r(x) .
$$

Therefore, on the set where $\Phi(x, y)=0$, we have

$$
D_{d}=-r(x)^{2}+r(x)(x-y) \cdot \nabla r(x) .
$$

We rewrite this as

$$
D_{d}=r(x)^{2}\left(r(x)^{-1}(x-y) \cdot \nabla r(x)-1\right) .
$$

Note that $r(x)^{-1}(x-y)$ is a unit vector on the set where $\Phi(x, y)=0$, and that we are assuming that $|\nabla r(x)|<c<1$. Hence $\left|r(x)^{-1}(x-y) \cdot \nabla r(x)-1\right| \geq 1-c>0$, as claimed. This completes the proof. 


\section{Proof of Corollary 3.4}

Corollary 3.4 follows from the proof of Theorem 2.3 and the estimate, implicit in [8] (see also [27]), that if

$$
\mathcal{A}_{\Gamma} f(x)=\sup _{1<t<2}\left|\int_{\Gamma} f(x-t y) d \sigma(y)\right|,
$$

where $\Gamma$ is a smooth hypersurface with non-vanishing Gaussian curvature and $d \sigma$ is the Lebesgue measure on $\Gamma$, then

$$
\left\|\mathcal{A}_{\Gamma} f_{j}\right\|_{L^{2}\left(\mathbb{R}^{d}\right)} \lesssim 2^{-\frac{j(d-2)}{2}}\left\|f_{j}\right\|_{L^{2}\left(\mathbb{R}^{d}\right)},
$$

where $f_{j}$ is the Littlewood-Paley piece of $f$ defined as in Section 4 (after (4.6)). Since the averaging operator $T$ associated with $\mathcal{G}$ is dominated by the maximal operator $\mathcal{A}_{\Gamma}$, it follows that $\left\|T f_{j}\right\|_{2} \lesssim 2^{-\frac{j(d-2)}{2}}\left\|f_{j}\right\|_{2}$. We now plug this directly into (4.9) in the proof of Theorem 2.3 (instead of using the assumption (2.1)). Since this is the only place where (2.1) was used, and all remaining assumptions of the theorem are satisfied, we obtain the corollary.

We now prove (7.1). We have

$$
\mathcal{A}_{\Gamma} f(x):=\sup _{1<t<2}\left|\mathcal{A}_{t} f(x)\right|, \quad \text { where } A_{t} f(x):=\int f(x-t y) d \sigma(y) .
$$

It suffices to show that

$$
\int \sup _{t}\left|A_{t} f_{j}(x)\right|^{2} d x \leq C\left\|f_{j}\right\|_{2}^{2} \cdot\left(2^{j}\right)^{-(d-2)} .
$$

In order to prove the claim, first observe that by the Fourier inversion formula we have

$$
A_{t} f_{j}(x)=\int_{|\xi| \sim 2^{j}} e^{2 \pi i x \cdot \xi} \widehat{f}_{j}(\xi) \widehat{\sigma}(t \xi) d \xi
$$

By the classical method of stationary phase (see, e.g., [22] and comments in [14]),

$$
|\widehat{\sigma}(\xi)| \leq C|\xi|^{-\frac{d-1}{2}},
$$

and, similarly,

$$
|\xi \cdot \nabla \widehat{\sigma}(\xi)| \leq C|\xi| \cdot|\xi|^{-\frac{d-1}{2}} .
$$

We need the following basic lemma, which is proved using the fundamental theorem of calculus and the Cauchy-Schwarz inequality. See, for example, 22], Chapter 2 .

Lemma 7.1. Let $F$ be a continuously differentiable function. Then

$$
\sup _{t \in[1,2]}|F(t)|^{2} \leq|F(1)|^{2}+2\left(\int_{1}^{2}|F(t)|^{2} d t\right)^{\frac{1}{2}} \cdot\left(\int_{1}^{2}\left|F^{\prime}(t)\right|^{2} d t\right)^{\frac{1}{2}} .
$$


We now apply Lemma (7.1) to $F(t)=A_{t} f_{j}(x)$ and use Cauchy-Schwarz to see that

$$
\begin{gathered}
\int \sup _{t \in[1,2]}\left|A_{t} f_{j}(x)\right|^{2} d x \leq \int\left|A_{1} f_{j}(x)\right|^{2} d x \\
+\left(\iint_{1}^{2}\left|A_{t} f_{j}(x)\right|^{2} d x d t\right)^{\frac{1}{2}} \cdot\left(\iint_{1}^{2}\left|\frac{d}{d t} A_{t} f_{j}(x)\right|^{2} d x d t\right)^{\frac{1}{2}} \\
=I+I I \cdot I I I .
\end{gathered}
$$

By Plancherel and (7.4),

$$
I \leq C|| f_{j} \|_{2}^{2} \cdot 2^{-j(d-1)}
$$

Applying Plancherel once again,

$$
I I \leq C\left\|f_{j}\right\|_{2} \cdot 2^{-j \frac{d-1}{2}} .
$$

Since $\frac{d}{d t} \widehat{\sigma}(t \xi)=\xi \cdot \widehat{\sigma}(t \xi)$, Plancherel and (7.5) imply that

$$
I I I \leq C\left\|f_{j}\right\|_{2} \cdot 2^{j} \cdot 2^{-j \frac{d-1}{2}} .
$$

Combining these estimates we see that

$$
\left|A_{t} f_{j}(x)\right|^{2} \leq C\left\|f_{j}\right\|_{2}^{2} \cdot 2^{j} \cdot\left(2^{j}\right)^{-(d-1)},
$$

where $C$ does not depend on $t$. It follows that

$$
\left(\sup _{t \in[1,2]}\left|A_{t} f_{j}(x)\right|^{2} d x\right)^{\frac{1}{2}} \leq C\left\|f_{j}\right\|_{2} \cdot\left(2^{j}\right)^{-\frac{d-2}{2}},
$$

which is what we wanted to prove.

\section{Proof of Corollary 3.5}

Recall that the Minkowski functional of $K$ is the unique function $\phi_{K}: \mathbb{R}^{d} \rightarrow$ $[0, \infty)$ which is homogeneous of degree 1 and equal to 1 on $\Gamma$. Let $f(x)$ be a smooth function on $\mathbb{R}^{d}$, non-vanishing except on $\Gamma$, such that $f(x)=1-\phi_{K}(x)$ for $\frac{a m}{2}<|x|<2 b M$. We would like to apply Corollary 3.4 with $\Phi(x, y)=f\left(\frac{y-x}{r(x)}\right)$. It is clear from the definition that all the assumptions are satisfied, except for (i), which needs to be verified. By explicit computation, we have for all $x, y$ with $|\Phi(x, y)|<\epsilon$ small enough,

$$
\begin{gathered}
\nabla_{y} \Phi(x, y)=-\frac{1}{r(x)} \nabla f\left(\frac{y-x}{r(x)}\right), \\
\nabla_{x} \Phi(x, y)=\frac{1}{r(x)}\left(\nabla f\left(\frac{y-x}{r(x)}\right)+\left(\frac{y-x}{r(x)} \cdot \nabla f\left(\frac{y-x}{r(x)}\right)\right) \nabla r(x)\right) .
\end{gathered}
$$

(8.1) is clearly bounded away from 0 as required, by the definition of $f$. Turning to (8.2), we note that since $r$ is bounded from above and below, and since $\frac{y-x}{r(x)} \in \Gamma$ whenever $\Phi(x, y)=0$, it suffices to prove that the quantity

$$
\nabla f(u)+(u \cdot \nabla f(u)) \nabla r(x)
$$


has norm bounded away from 0 if $u \in \Gamma$. Using the definition of $f$, and computing its directional derivative in the direction of $u$ at $u \in \Gamma$, we get $u \cdot \nabla f(u)=1$ on $\Gamma$. We also get that for $u \in \Gamma$,

$$
|\nabla f(u)|=\frac{1}{|u| \cos \theta},
$$

where $\theta$ is the angle between $u$ and the outward normal vector to $\Gamma$ at $u$. But the right side is also equal to the distance between the origin and the tangent line to $\Gamma$ at $u$. By the convexity of $K$, this is minimized when $|u|=m$ and maximixed when $|u|=M$, and at those points we have $\cos \theta=1$. Thus $m \leq|\nabla f(u)| \leq M$. It follows that for $u \in \Gamma$,

$$
|\underline{(8.3)}| \geq|| \nabla f(u)|-r(x)|>\epsilon_{0}>0
$$

if one of (3.8), (3.9) holds. By continuity, a similar estimate (with $\epsilon_{0}$ replaced by $\epsilon_{0} / 2$ ) holds on a neighbourhood of $\Gamma$. This completes the verification of (i) and hence proves the corollary.

\section{REFERENCES}

[1] B. Aronov, M. Sharir, Cutting circles into pseudo-segments and improved bounds for incidences, Discrete Comp. Geom. 28 (2002), 475-490. MR.1949895 (2004i:52011)

[2] B. Aronov, V. Koltun, M. Sharir. Incidences between points and circles in three and higher dimensions. Discr. Comp. Geom. 33 (2005), 185-206. MR2121292 (2005k:52027)

[3] B. Aronov, J. Pach, M. Sharir, G. Tardos. Distinct distances in three and higher dimensions. Combin. Probab. Comput. 13 (2004) 283-293. MR2056399 (2005b:52017)

[4] Michael Christ, Quasi-extremals for a Radon-like transform, preprint, 2006.

[5] K. Clarkson, H. Edelsbrunner, L. Guibas, M. Sharir, E. Welzl. Combinatorial complexity bounds for arrangements of curves and spheres. Discrete Comput. Geom. 5 (1990) 99-160. MR:1032370 (91f:52021)

[6] G. Elekes, Cs. Tóth: Incidences of not too degenerate hyperplanes, preprint, 2005.

[7] K. J. Falconer, On the Hausdorff dimensions of distance sets. Mathematika 32 (1985) 206212. MR $834490(87 \mathrm{j}: 28008)$

[8] A. Greenleaf, Principal curvature in harmonic analysis. Indiana Math. J. 30 (1981) 519-537. MR620265 (84i:42030)

[9] L. Hörmander, The analysis of linear partial differential operators IV. Springer-Verlag, Berlin, 1985. MR0781537 (87d:35002b)

[10] A. Iosevich, Fourier analysis and geometric combinatorics. To appear in the Birkhauser volume dedicated to the annual Padova lectures in analysis, 2004.

[11] A. Iosevich, S. Hofmann, Circular averages and Falconer/Erdös distance conjecture in the plane for random metrics, Proc. Amer. Math. Soc. 133 (2005), 133-143. MR2085162 (2005k:42031)

[12] A. Iosevich, I. Łaba, K-distance sets, Falconer conjecture, and discrete analogs, Integers: Electronic Journal of Combinatorial Number Theory, 5 (2005), \#A08 (hardcopy in: Topics in Combinatorial Number Theory: Proceedings of the Integers Conference 2003 in Honor of Tom Brown, DIMATIA, ITI Series, vol. 261). MR2192086 (2006i:42033)

[13] A. Iosevich, M. Rudnev, Distance measures for well-distributed sets, Discrete Comput. Geom. 38 (2007), 61-80. MR2322116 (2008m:52038)

[14] A. Iosevich and E. Sawyer, Maximal averages over surfaces, Advances in Mathematics, 132, (1997), 46-119. MR:1488239 (99b:42023)

[15] I. Łaba, J. Solymosi, Incidence theorems for pseudoflats, Discrete Comput. Geom. 37 (2007), 163-174. MR2295051 (2008e:52015)

[16] J. Pach, M. Sharir, On the number of incidences between points and curves, Combinatorics, Probability and Computing 7 (1998), 121-127. MR1611057(99b:52037)

[17] J. Pach, M. Sharir, Geometric incidences, in: Towards a Theory of Geometric Graphs (J. Pach, ed.), Contemporary Mathematics, vol. 342, Amer. Math. Soc., Providence, RI, 2004. MR:2065247 (2004m:05004) 
[18] D. Phong, E. M. Stein, Hilbert integrals, singular integrals, and Radon transforms I. Acta Math. 157 (1986) 99-157. MR857680 (88i:42028a)

[19] W. Schlag. On continuum incidence problems related to harmonic analysis. J. Funct. Anal. 201 (2003) 480-521. MR.1986697 (2004k:43009)

[20] M. Sharir. On joints in arrangements of lines in space and related problems. J. Comb. Theory A 67 (1994) 89-99. MR 1280600 (96c:52023)

[21] M. Sharir, E. Welzl. Point-line incidences in space. Combinatorics, Probability and Computing 13 (2004) 203-220. MR2047237 (2005b:52033)

[22] C. D. Sogge. Fourier integral in classical analysis. Cambridge Tracts in Mathematics 105, Cambridge Univ. Press, 1993. MR.1205579 (94c:35178)

[23] J. Solymosi and Cs. Tóth, Distinct distances in the plane, Discrete Comput. Geometry 25 (2001), 629-634. MR:1838423 (2002c:52020)

[24] J. Solymosi and Cs. Tóth, Distinct distances in homogeneous sets in Euclidean space, Discrete Comput. Geom. 35 (2006), 537-549. MR2225673 (2007c:52020)

[25] J. Solymosi and V. Vu, Distinct distances in high dimensional homogeneous sets in: Towards a Theory of Geometric Graphs (J. Pach, ed.), Contemporary Mathematics, vol. 342, Amer. Math. Soc., Providence, RI, 2004. MR2065269 (2005m:52026)

[26] J. Spencer, E. Szemerédi, W. Trotter, Unit distances in the Euclidean plane, in: Graph Theory and Combinatorics, B. Bollobás, ed., Academic Press, New York, 1984, 293-303. MR7777185 (86m:52015)

[27] E. M. Stein. Harmonic Analysis. Princeton University Press, 1993. MR1232192 (95c:42002)

[28] L. Székely. Crossing numbers and hard Erdös problems in discrete geometry. Combinatorics, Probability, and Computing 6 (1997) 353-358. MR1464571(98h:52030)

[29] E. Szemerédi, W. Trotter. Extremal problems in discrete geometry. Combinatorica 3 (1983) 381-392. MR729791 (85j:52014)

[30] T. Wolff. Recent work connected with the Kakeya problem. Prospects in Mathematics. Amer. Math. Soc., Providence, RI, 1999, 129-162. MR1660476 (2000d:42010)

[31] T. Wolff. Lectures on Harmonic Analysis. I. Łaba and C. Shubin, eds.. University Lecture Series, vol. 29, American Mathematical Society, 2003. MR2003254 (2004e:42002)

Department of Mathematics, University of Missouri, 201 MSB, Columbia, Missouri 65211-4100

Department of Mathematics, Princeton University, Washington Road, Fine Hall, Princeton, New Jersey 08544

Department of Mathematics, University of British Columbia, Vancouver, British Columbia, Canada V6T 1Z2 